\title{
Support Vector Machine Optimization Based On Magnetic Bacteria Optimization Algorithm
}

\author{
Ce Yang and Zhaofeng Chen \\ Naval Academy of Armament \\ xyangcej@gmail.com,zhaofeng1981225@126.com
}

\begin{abstract}
Classification performance of support vector machine (SVM) will be influenced by its model parameters. For this problem, a new method named magnetic bacteria optimization algorithm (MBOA) that optimizes the parameters of SVM is proposed. It is tested by the UCI standard data sets and compared with the other optimization algorithms, such as particle swarm optimization (PSO). Experimental results show that the MBOA can optimize the parameters of SVM well and has better performance than the compared algorithms.
\end{abstract}

Keywords: Magnetic Bacteria Optimization Algorithm; Support Vector Machine; Optimization; Classification Performance

\section{Introduction}

SVM is an excellent machine learning tool. In recent years, it has made a breakthrough in theory and algorithms. It is considered as a very popular and successful example on machine learning and pattern recognition field. The SVM largely overcomes many traditional classification problems, such as "curse of dimensionality" and "overlearning"[1].

When SVM is used for solving practical problems, it needs to select the model parameters. The parameters directly determine the training effectiveness and application efficiency. So how to choose the parameters to get the best optimal classification performance is the main problem of SVM [2]. The SVM involves kernel function model, kernel function parameters and the penalty factor C. It has been proved that there exists multimodal function relationship between SVM classification accuracy and these parameters. There are a lot of intelligence optimization techniques inspired by biologic phenomena, such as Particle Swarm Optimization (PSO), Genetic Algorithm (GA), Artificial Bee Colony (ABC), Biogeography Based Optimization (BBO) and so on.

MBOA is a new intelligent optimization algorithm [3]. The algorithm is inspired by the biological behavior of magnetic bacteria, which can move along the magnetic field lines. It matches the optimization problem with magnetic bacterial biological characteristics, finding the optimal solution. The MBOA has been tested by standard functions. Lots of experiments show that the optimization algorithm can effectively solve the optimization problems. MBOA shows better performance and good potential ability in solving optimization problems. It has been improved into several new variants of MBOA [4-9].

In this paper, we use the MBOA to optimize the parameters of SVM. Then the optimized SVM is used for classification and tested by standard tests with UCI data. It is compared with other typical optimization algorithms. 


\section{Support Vector Machine Parameters}

The kernel function of SVM is one of the most important problems. For nonlinear problems, the data is usually mapped from low dimensional space to high dimensional space, and construct a linear discriminate function in the high-dimensional feature space to achieve the nonlinear discriminate function in original space[3]. Different kernel functions mean different mapping. Kernel function is operated in the input space rather than in high-dimensional space, so it doesn't need to calculate the inner product in the feature space. The kernel functions used in SVM mainly have four types: linear, polynomial, RBF kernel, and Sigmoid kernel[10].

Under the common sense, RBF Gaussian radial basis function is a priority target, so here choosing RBF kernel function describe the problem, other types of kernel functions will also be applied to.

There are two parameters which need to be optimized in SVM. One is the penalty factor $C$, another is nuclear parameter $\mathrm{g}$. The role of penalty factor $C$ is to compromise the complexity of data space and generalization performance. It reflects the degree of error tolerance. When $C$ is smaller, the ability of SVM punishing training set is weak, so the empirical risk is great and the accuracy is greatly lost. At the same time, the generalization performance of SVM will be enhanced, which is called "less learning" problem. When $C$ is larger, the accuracy is improved, but it also increases the complexity of the model, and the generalization performance is reduced, which is called "over learning" problem[11]. Thus appropriate selection of penalty factor $C$, will make the SVM balance between data fitting and generalization performance. Thus it can obtain the best classification performance.

\section{Principle of Magnetic Bacteria Optimization Algorithm}

\subsection{Biological Basis}

In nature, there is a kind of polyphyletic group of prokaryotes that can orient and swim along magnetic field lines. They are called magnetotactic bacteria (MTB) [13]. Motility is an essential ability by which most bacteria can find optimal localities in their environment to maximize their substrate or energy uptake. Bacterial motility can be modulated via sensing energy levels within cells and of chemical signals in the environment. A striking property of magnetotactic bacteria is their ability to orient and propel themselves along geomagnetic field lines (magnetotaxis) in the earth magnetic field.

Magnetotactic bacteria occur widely in natural sediments from both marine and freshwater habitats. Magnetosomes (MTS) are magnetite colloids, compounded by membrane-bounded magnetite particles, produced from intracellular. MTS is constituted by colloidal size particles with a fairly narrow size distribution. They are mineral particles with specific crystallographic and enveloping membrane, which are typically arranged in the form of one or several chains and impart a permanent magnetic dipole moment to the bacterium [14]. So the compass of magnetotactic bacteria is made up of a chain of magnetosomes which keeps the long axis of the bacteria parallel to the geomagnetic field direction [14]. Bacteria can find the best oxygen concentration and the redox potential in the water column or at the contact areas of sediment and water. This behavior can increase the efficiency with which such bacteria can find their optimal oxygen concentrations or redox potentials at sedimentwater interfaces or in water columns.

Now we know that the magnetosomes play important role in regulating the movement of magnetotactic bacteria. The size, shape and amount of magnetosomes have important effect on the ability and motion efficiency of magnetotactic bacteria in the interaction with magnetic field. In fact, the magnetotactic bacteria have evolved to be adaptive to the magnetic field. Only those magnetotactic bacteria with magnetosomes, which can make 
magnetic field lines bend in some of the magnetosomes to minimize their magnetostatic energy, can survive in nature. Each magnetosome can produce moment. In a MTB, the total magnetic moments is the sum of all moments of magnetosomes. The magnetotactic bacteria need to produce the magnetosome moments which can minimize their magnetostatic energy[14]. And the optimization process of minimizing their magnetostatic energy by producing the magnetosome moments is considered as a process of solving optimization problems[5].

\subsection{Model of MBOA}

Multi cells with magnetosomes interact each other to be adaptive to the magnetic field. Each cell has mount of energy decided by the magnetic field and its total moments. Suppose $E$ as the energy of a cell, we ignore the orientation of magnetic field, and have

$$
E=M \cdot B==M \times B \times \cos \theta
$$

Where $M$ is the total moments of a magnetosome, $B$ is the magnetic field of the magnetosome, and $\theta$ is the angle between $M$ and $B$.

When the MBOA runs to solve a problem, it corresponds to the process of producing MTS to be adaptive to magnetic field. It needs to regulate the moments of each MTS, just like producing feasible solutions. MBOA begins by computing the distance between two randomly selected cells, followed by calculating interaction energy. Then, the moments of magnetisms are calculated. The MTS of a cell are generated based on the obtained moments, which is called MTS generation in the algorithm. After the generation, they will expand in size in order to obtain better moments. This is called MTS expanding in the algorithm. Finally, some MTS with worse moments will be replaced, which is called MTS replacement.

MBOA obtains the optimal solution by regulating the moments of cells continually by the process of MTS generation, MTS expanding and MTS replacement. When the MBOA obtains the optimal solution, it corresponds to the state that when the moments of all cells are oriented in the geomagnetic field.

The MBOA includes the following steps:

\section{Initialization}

The initial population is filled with $N$ number of randomly generated $L$ dimensional real-valued vectors. Let $X_{i}=\left(x_{i 1}, x_{i 2}, \ldots, x_{i L}\right)$ represents the $i$ th cell (for $t=0)$ initialized randomly. Then each MTS in a cell is generated as follows:

$$
x_{i k}=x_{\text {min }}+\operatorname{rand} *\left(x_{\text {max }}-x_{\text {min }}\right)
$$

where $i=1,2, \ldots, N, k=1,2, \ldots, L \cdot x_{\max }$ and $x_{\min }$ are upper and lower bounds for the $x_{i k}$, respectively. $L$ is the number of dimension. rand is a random number between 0 and 1 .

2. Interaction energy calculation

In this step, we should follow the equation (7) to calculate the interaction energy between two cells.

But for producing diverse solutions in the algorithm, we do not follow the biology strictly. At first, we suppose $X_{r 1}, X_{r 2}(r 1, r 2 \in(1, \ldots, i, \ldots, N))$ as the two cells in the population, respectively. $t$ is the number of generation. $D$ is defined as the following normalization function.

$$
D\left(X_{r 1}, X_{r 2}\right)=\text { norm }\left\|X_{r 1}-X_{r 2}\right\|
$$


where $\operatorname{norm}\left(D_{i}\right)$ is the Euclidean length of vector $D_{i}\left(d_{i 1}, d_{i 2}, \ldots d_{i j}, \ldots, d_{i L}\right)$.

The interaction energy $E_{i}\left(e_{i 1}, e_{i 2}, \ldots, e_{i L}\right)$ is calculated as follows:

$$
e_{i j}(t)=\left(\frac{1}{1+N * d_{i j}(t)+L * d_{i j}(t)}\right)^{3}
$$

where $d_{i j}$ is the distance between two dimensions $x_{r 1, j}, x_{r 2, j}$ of $X_{r 1}, X_{r 2}$.

According to the process of the energy production according to (11) and (12), much more diverse solutions can be obtained for the algorithm.

3. MTS generation

According to (7), for simplification, here we suppose $\cos \theta=1$, we get

$$
M_{i}(t)=\frac{E_{i}(t)}{B}
$$

Suppose $M_{i}=\left(m_{i 1}, m_{i 2}, \ldots, m_{i L}\right)$, the MTSs are produced and their moments are regulated as follows:

$$
x_{i q}(t)=x_{p q}(t)+m_{p q}(t) * \text { rand }
$$

where $p, q$ are randomly selected from $\{1,2, \ldots, L\}$. rand is a random number between 0 and 1 .

4. MTS expanding

After MTSs generation, the MTSs in the cell expand as follows:

We set a magnetic field strength $b=0.5$. If $r a n k>b$, the MTS in the cell are expanded and their moments are as follows:

$$
X_{i}(t+1)=X_{\text {best }}(t)+\left(X_{\text {best }}(t)-X_{i}(t)\right) * \operatorname{rand}(1, L)
$$

Otherwise, they are expanded as follows.

$$
X_{i}(t+1)=X_{i}(t)+\left(X_{\text {best }}(t)-X_{i}(t)\right) * \operatorname{rand}(1, L)
$$

Where $\operatorname{rand}(1, L)$ is randomly generated vector with $L$ dimensions. Each dimension value is between $(0,1)$.

5. MTS replacement

After the MTS expanding, some worse MTSs will be replaced and their moments are changed by the following way:

$$
X_{i}(t+1)=m_{p q}(t) *(\operatorname{rand}(1, L)-1) * \operatorname{rand}(1, L)
$$

In general, the generation number is set as the stopping condition.

Generally, MBOA can be described as follows:

I. Define the simple bounds, determination of algorithm parameters.

II. Initialization: Randomly create the initial population in the search space.

III. while stop criteria is not met

for $=1: \mathrm{N}$

Calculate interaction distance according to(3)

end for

for $=1: \mathrm{N}$

for $=1: \mathrm{N}$

Calculate interaction energy according to (4)

end for

Obtain moments according to (5)

for $=1: \mathrm{N}$ 


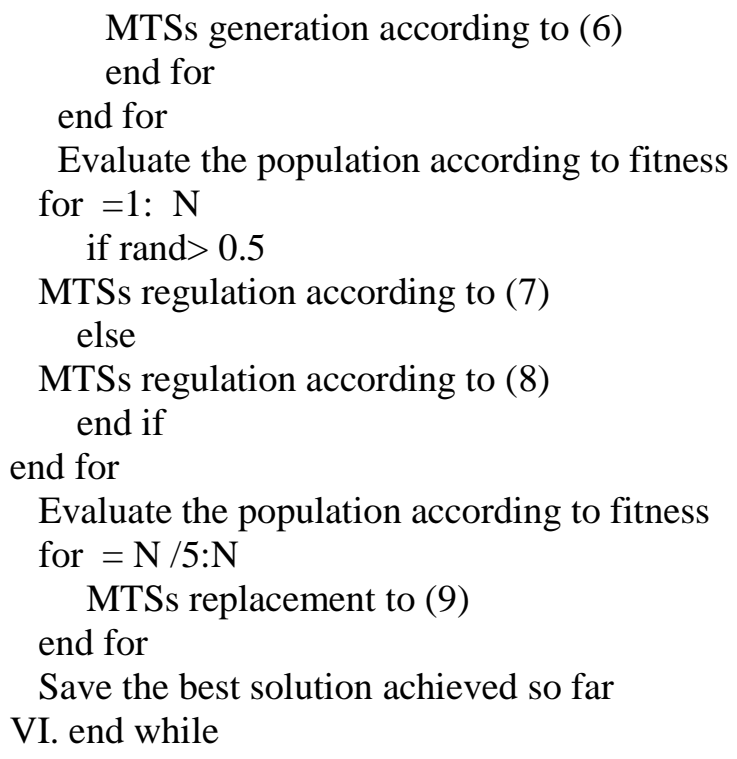

\section{MBOA Optimize SVM}

In this section, we use MBOA to optimize the parameters of penalty factor $C$ and kernel parameter $g$. The other specific parameters of algorithms are given below:

1 Set the size of the population, the number of iterations, the earth magnetic field strength, and parameters $C$ and $g$.

2 Set the fitness function. To achieve higher classification accuracy and improve the classification performance of SVM, the classification accuracy is set as the fitness function.

In all experiments, all algorithms are the basic ones without any improvement. And their parameters are set as follows:

Population size is 20 . Generation is 20 . Search range of optimize parameters $C$ and $g$ is set as [0.1,1000].

MBOA setting: In the MBOA, the magnetic field $B$ is set as 3 .

GA Settings: In our experiments, we employ a real number coded standard GA having evaluation, fitness scaling, seeded selection, random selection, crossover, mutation and elite units. Single point crossover operation with the rate of 0.8 is employed. Mutation operation restores genetic diversity lost during the application of reproduction and crossover. Mutation rate in our experiments is 0.01 .

DE Settings: In DE, $F$ is a real constant which affects the differential variation between two solutions and set to 0.5 in our experiments. Value of crossover rate, which controls the change of the diversity of the population, is chosen to be 0.9 .

PSO Settings: In our experiments cognitive and social components are both set to 2 . Inertia weight, which determines how the previous velocity of the particle influences the velocity in the next iteration, is 0.8 .

ABC Settings: Limit=100, which is a control parameter in order to abandon the food source.

BBO Settings: For BBO, we use the following parameters: habitat modification probability is 1 , immigration probability bounds per gene are [0,1], step size for numerical integration of probabilities, maximum immigration and migration rates for each island are 1 , and mutation probability is 0 .

UCI standard data is used as test data[16]. Some data sets are showed in Table 1.

The method is tested by the UCI standard data sets and compared with the other optimization algorithms. For the SVM, we use libsvm software toolkit. 
Table 1 UCI Datasets

\begin{tabular}{ccccc}
\hline UCIstandard & Category & Dimension & Train & Test \\
\hline Breast & 2 & 9 & 200 & 77 \\
Heart & 3 & 13 & 190 & 80 \\
Iris & 3 & 4 & 90 & 60 \\
Vehicle & 4 & 18 & 400 & 446 \\
\hline
\end{tabular}

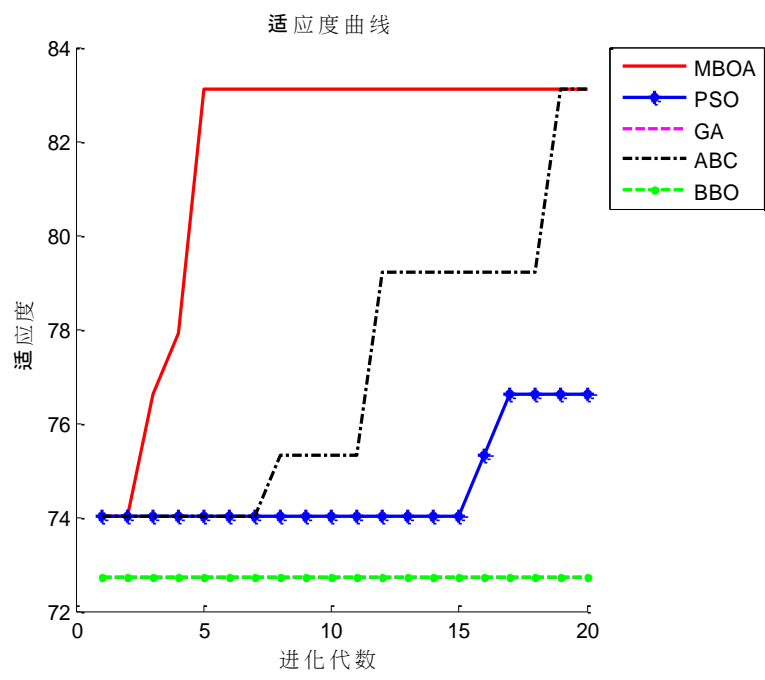

Figure 1: Fitness Curve Corresponding To Each Algorithm Of Dataset Breast

Table 1: The Optimal Value Of Dataset Breast

\begin{tabular}{cccccc}
\hline $\begin{array}{c}\text { Algorith } \\
\mathrm{m}\end{array}$ & $\begin{array}{c}\text { MBO } \\
\mathrm{A}\end{array}$ & PSO & GA & ABC & BBO \\
\hline Fitness & 83.12 & 76.62 & 72.73 & 83.12 & 72.73 \\
Best C & 1.25 & 72.26 & 10.58 & 1.21 & 890.15 \\
Best g & 0.10 & 0.10 & 725.56 & 0.10 & 987.90 \\
\hline
\end{tabular}

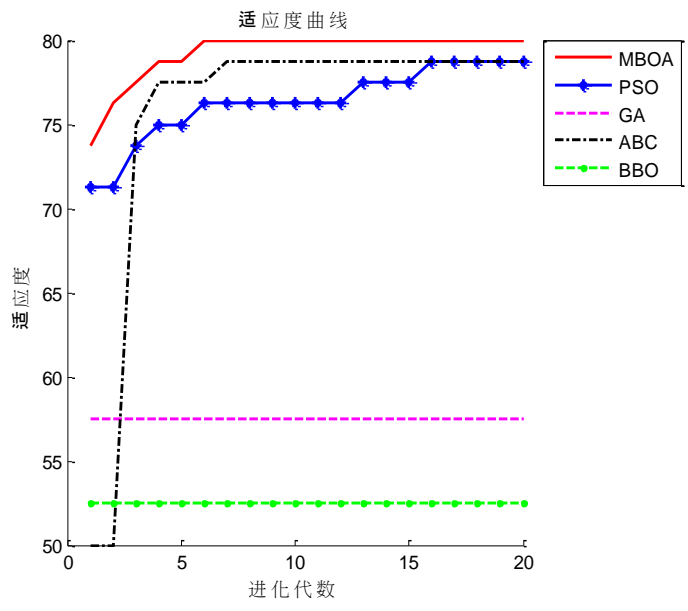

Figure 2: Fitness Curve Corresponding To Each Algorithm of Dataset Heart 
Table 2: The Optimal Value Of Dataset Heart

\begin{tabular}{cclllc}
\hline Algorith & \multicolumn{1}{c}{ MBO } & PSO & GA & ABC & \multicolumn{2}{c}{ BB } \\
$\mathrm{m}$ & A & & & & \multicolumn{2}{c}{ O } \\
\hline Fitness & 80.00 & 77.50 & 61.75 & 79.75 & 60. \\
Best C & 6.75 & 129.8 & 963.2 & 121.3 & 00 \\
Best g & 0.10 & 0.10 & 1 & 0.10 & 87 \\
& & & & & 8.1 \\
& & & & & 17 \\
\hline
\end{tabular}

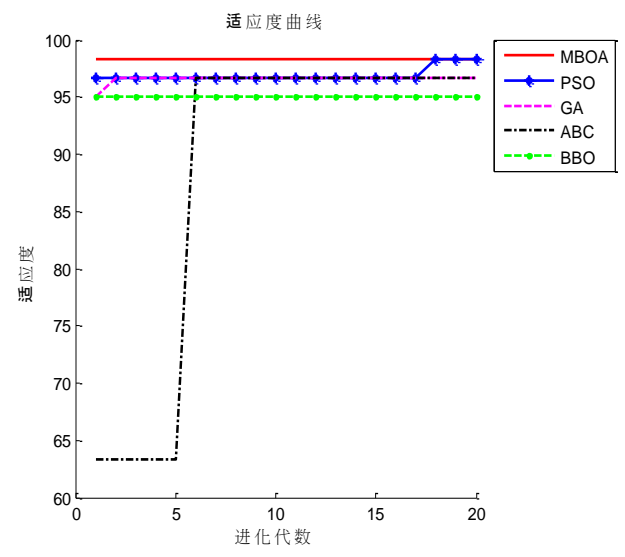

Figure 3: Fitness Curve Corresponding to Each Algorithm of Dataset Iris

Table 3: The Optimal Value Of Dataset Iris

\begin{tabular}{ccclccc}
\hline $\begin{array}{c}\text { Algorit } \\
\text { hm }\end{array}$ & \multicolumn{1}{c}{ MBO } & PSO & GA & ABC & \multicolumn{2}{c}{ BB } \\
\hline Fitness & 98.33 & 98.33 & 96.67 & 96.67 & 95 \\
Best C & 22.69 & 24.34 & 1 & 899.8 & 799 \\
Best g & 0.10 & 0.10 & 6.95 & 7 & & \multicolumn{2}{c}{.74} \\
& & & & 0.10 & \multicolumn{2}{c}{6.1} \\
& & & & & \multicolumn{2}{c}{6} \\
\hline
\end{tabular}

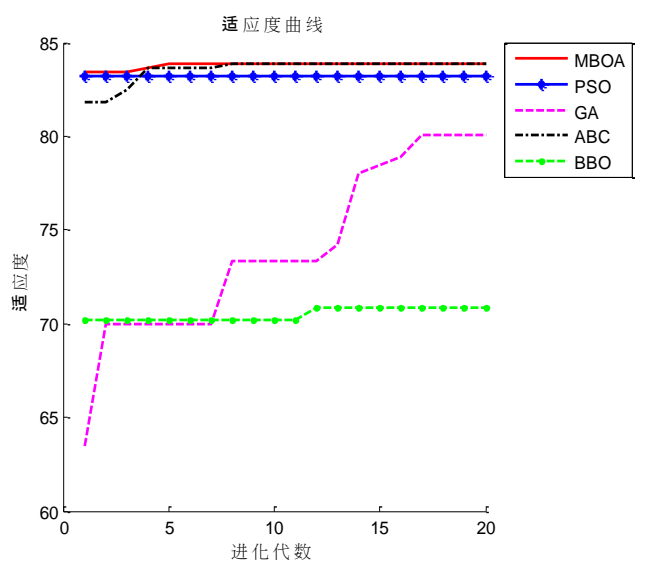

Figure 4: Fitness Curve Corresponding To Each Algorithm of Dataset Vehicle 
Table 4: The Optimal Value Of Dataset Vehicle

\begin{tabular}{crllllc}
\hline Algorit & \multicolumn{2}{c}{ MBO } & PSO & GA & ABC & \multicolumn{2}{c}{ BB } \\
$\mathrm{hm}$ & \multicolumn{1}{c}{ A } & & & \multicolumn{3}{c}{ O } \\
\hline Fitness & 83.86 & 83.18 & 80.04 & 83.86 & \multicolumn{2}{c}{70.8} \\
Best C & 805.3 & 776.6 & 86 & 762.9 & 5 & \\
Best g & 6 & & 0.10 & 1 & 8 & \multicolumn{2}{c}{794.} \\
& 0.13 & & & 0.10 & \multicolumn{2}{c}{54} \\
& & & & & \multicolumn{2}{c}{12} \\
\hline
\end{tabular}

From Figure 1, 2, 3 and 4, Table 1, 2, 3 and 4, it can be seen that, for breast, heart datasets, MBOA has better classification performance than PSO and ABC. For the datasets Iris and vehicle, all five algorithms have good performance. The difference dataset itself, including dimensions, categories, will affect the classification results. But MBOA have the fastest convergence speed than all the compared algorithms. Fast convergence and better optimal solution of MBOA indicate MBOA can effectively solve optimization problems.

\section{Conclusion}

The parameters of SVM are crucial factors for classification performance, To improve classification accuracy, we use MBOA to optimize SVM parameters. The classification accuracy is used as fitness function. It optimizes the penalty factor $\mathrm{C}$ and parameter $\mathrm{g}$. The results showed that MBOA can quickly converge on the same number of iterations. At the same time, it achieved higher classification accuracy. MBOA is more competitive compared to other algorithms. This novel optimization algorithm based on bio-inspired can become a new way to solve the classification problem.

\section{References}

[1] J. J. Li, L.H. Zhao and A. X. Wang. Pattern Recognition. Beijing: Electronic Industry Press, (2010) .

[2] L.Lu and T. Y. Wang. Support Vector Machine Optimization Based on Artificial Bee Colony Algorithm. Journal of Tianjin University, (2011).

[3] H.W. Mo and L.F. Xu. Magnetotactic Bacteria Algorithm for Function Optimization. Journal of Software Engineering and Applications, 5: 66-69 (2013).

[4] H.W. Mo and L.F. Xu. Magnetotactic bacteria optimization algorithm for multimodal optimization. IEEE Symposium on Swarm Intelligence (SIS), (2013)December23-25Sinpore.

[5] H.W. Mo, LL..Liu, L.F. Xu and Y.Y. Zhao. Performance research on magnetotactic bacteria optimization algorithm based on the best individual. The sixth International Conference on Bio-inspired Computing(BICTA2014), (2014)October16-18:Wuhan,China.

[6] H.W. Mo and M.J.Geng. Magnetotactic bacteria optimization algorithm based on best-rand scheme. 6th Naturei and Biologically Inspired Computing. Porto Portugal. 59-64(2014).

[7] H.W.Mo and L.L. Liu. Magnetotactic bacteria optimization algorithm based on best-target scheme. International Conference On Nature Computing and Fuzzy Knowledge, (2014)August22-25:Xiamen, China.

[8] H.W.Mo and L.L. Liu,L.F. Xu, A power spectrum optimization algorithm inspired by magnetotactic bacteria. Neural Computing and Applications, 25(7-8): 1823-1844 (2014).

[9] H.W. Mo, L.L. Liu and M.J. Geng. A new magnetotactic bacteria optimization algorithm based on moment migration. 2014 International Conference on Swarm Intelligence. (2014) October16-18: Hefei,China.

[10] H.Y. Li. Support vector machine kernel function study . Chongqing University, (2009).

[11] Y. M. Zhao. Pattern Recognition. Shanghai Jiaotong University Press, Shanghai (2013).

[12] D. Faivre, D. Schüler. Magnetotactic bacteria and magnetosomes. Chemical, Reviews, 108(11): 48754898(2008).

[13] A. P. Philipse and D. Maas. Magnetic colloids from magnetotactic bacteria: chain formation and colloidal stability. Langmuir, 18(25): 9977-9984(2002).

[14] http://archive.ics.uci.edu/ml/ 